\title{
Modélisation physique de l'impact du disponible sédimentaire sur la morphologie des structures sédimentaires en courant seul
}

\author{
Mélanie VAH ${ }^{1}$, Armelle JARNO ${ }^{1}$, Sophie LE BOT ${ }^{2}$, François MARIN ${ }^{1}$ \\ 1. Université de Le Havre Normandie, UMR 6294 CNRS - LOMC, \\ 53 rue de Prony, 76600 Le Havre, France. \\ melanie.vah@ac-rouen.fr \\ 2. Université de Rouen Normandie, UFR Sciences et Techniques, UMR 6143 CNRS - \\ $\mathrm{M} 2 \mathrm{C}$, \\ Bâtiment Blondel, Place Emile Blondel, 76821 Mont Saint Aignan, France.
}

\section{Résumé :}

Les expériences ont été menées dans un canal à courant à fond horizontal. Le disponible sédimentaire initial varie dans une gamme allant d'extrêmement limité à illimité. Une recirculation des sédiments est assurée de manière à conserver un disponible constant tout au long de chaque test. Dans le cas d'un sable moyen bien trié, l'étude de l'évolution des longueurs d'onde des structures sédimentaires à l'équilibre en fonction du disponible sédimentaire pour différentes conditions hydrodynamiques initiales est proposée. Une augmentation de la longueur d'onde est constatée quand le disponible sédimentaire augmente. L'irrégularité des structures sédimentaires est aussi discutée. Les longueurs d'onde à l'équilibre en disponible infini sont comparées avec la littérature et sont en bon accord sauf dans le cas de formes de petite échelle surimposées à des structures de plus grande échelle.

\section{Mots-clés :}

Structures sédimentaires, Disponible sédimentaire limité, Dynamique sédimentaire, Modélisation physique

\section{Introduction}

Les structures sédimentaires jouent un rôle majeur sur le frottement au fond, les conditions d'écoulement et le transport sédimentaire. Ces structures sédimentaires sont majoritairement discriminées selon leurs dimensions, les conditions d'écoulement ou encore le diamètre des sédiments (CHAMLEY, 1987 ; SOULSBY et al., 2012). Deux types de structures sédimentaires, transversales par rapport aux courants, sont communément considérés : les rides et les dunes, distinguées sur la base d'une limite de longueur d'onde de 0,6 m (ASHLEY, 1990), et respectivement dans l'ordre de grandeur du diamètre des grains et de la hauteur d'eau.

Les structures sédimentaires en disponible limité ne sont que très peu étudiées par rapport aux structures sédimentaires en disponible illimité alors que les conditions de disponible 


\section{Thème 2 - Dynamique sédimentaire}

sableux limité sont communes à la fois en rivière et en mer. Quelques études expérimentales ont été menées en canaux inclinés représentant des conditions fluviales (TUIJNDER et al., 2009 ; DREANO et al., 2010) et en canal à fond non incliné (VAH et al., 2020).

L'objectif de cette étude est de comparer qualitativement et quantitativement les longueurs d'ondes à l'équilibre de séries de tests réalisées avec un disponible sédimentaire variable. Les résultats sont comparés avec la littérature en disponible infini.

\section{Protocole et conditions expérimentales}

Les expériences sont menées dans un canal à courant à fond horizontal de $10 \mathrm{~m}$ de long et $0,49 \mathrm{~m}$ de large (voir figure 1). La recirculation de l'eau est obtenue grâce une pompe centrifuge. La hauteur d'eau au-dessus du lit sédimentaire initialement plat est $d=25 \mathrm{~cm}$. La longueur entre le nid d'abeille en entrée de canal, et les trappes de récupération des sédiments est de 7,4m. Le fond du canal est en PVC. Une opération de ponçage léger a été effectuée pour obtenir une surface micro-rugueuse homogène.

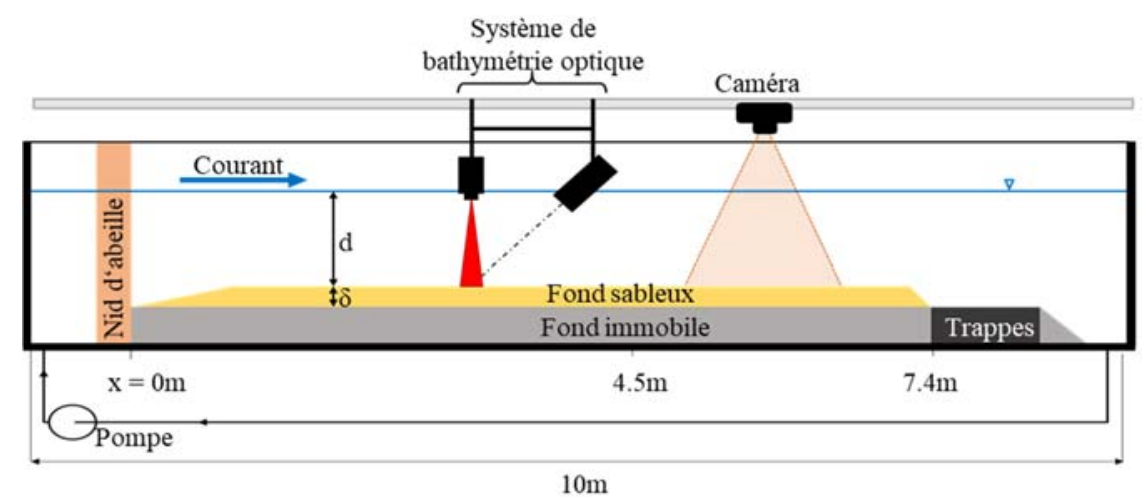

Figure 1. Schéma du canal expérimental avec les équipements de mesures.

Des profils de vitesse sans sédiments ont été effectués de manière préliminaire et ont montré un profil logarithmique établi caractéristique d'un écoulement turbulent à partir de 4,5 m à l'aval du nid d'abeilles.

Dans les expériences présentées, un sable naturel de carrière de densité relative $s=2,65$ et de diamètre médian $D_{50}=328 \mu \mathrm{m}$ est utilisé. Il est de granulométrie uniforme $\left(\sigma_{g}^{2}=1,5\right)$ selon le critère de Soulsby où $\sigma_{g}^{2}=D_{84} / D_{16}<2$ avec $D_{84}$ et $D_{16}$, les diamètres de grains tels que $84 \%$ et $16 \%$ des grains de l'échantillon en masse soient plus petits.

Le protocole de conduite des essais est le suivant :

- Le lit sédimentaire est aplani de manière à avoir une hauteur sédimentaire initiale $\delta$ (voir figure 1).

- Arrêts réguliers du courant : toutes les quinze minutes à une heure suivant la vitesse de l'écoulement au cours de la mise en équilibre du fond. 


\section{XVIèmes Journées Nationales Génie Côtier - Génie Civil \\ Le Havre 2020}

- Pendant les phases d'arrêt, photos haute résolution $(0,1 \mathrm{~mm} /$ pixel $)$ sur une zone de longueur $1,50 \mathrm{~m}$ située dans la zone d'écoulement établi $(5 \mathrm{~m}<\mathrm{x}<6,5 \mathrm{~m})$ et profils bathymétriques optiques (méthodologie décrite dans MARIN \& EZERSKY (2008) sur une longueur de $2,30 \mathrm{~m}(4,5 \mathrm{~m}<x<6,8 \mathrm{~m} ; 0,05 \mathrm{~mm} / \mathrm{pixel})$

- Etalement à l'amont du canal $(0 \mathrm{~m}<x<2,5 \mathrm{~m})$, au cours de chaque test, du sable issu des trappes pour assurer l'hypothèse d'un disponible sédimentaire quasi-constant.

Un post-traitement sous Matlab est réalisé afin de repérer la position des crêtes et des creux. La longueur d'onde est définie entre deux crêtes successives. La moyenne des longueurs d'onde est ensuite extraite à partir de l'ensemble des valeurs des longueurs d'onde.

Les tests ont été réalisés avec différentes épaisseurs sédimentaires : $\delta=0,1 \mathrm{~cm}, \delta=0,5 \mathrm{~cm}$, $\delta=1 \mathrm{~cm}, \delta=2 \mathrm{~cm}, \delta=3,5 \mathrm{~cm}$ et $\delta=5,5 \mathrm{~cm}$ permettant de varier les conditions de disponibilité en sédiments de 'très limitée' ( $\delta=1 \mathrm{~mm}$ ) à 'illimitée'. Les conditions expérimentales sont présentées dans le tableau 1. Pour le set M3, l'épaisseur $\delta=1 \mathrm{~mm}$ n'a pas été réalisée.

Tableau 1. Paramètres dimensionnels et adimensionnels.

\begin{tabular}{llll}
\hline & Set $\boldsymbol{M 1}$ & Set $\boldsymbol{M} 2$ & Set $\boldsymbol{M 3}$ \\
\hline$U[\mathrm{~m} / \mathrm{s}]$ & 0,35 & 0,45 & 0,55 \\
$u_{*}^{\prime}[\mathrm{m} / \mathrm{s}]$ & 0,016 & 0,021 & 0,026 \\
\hline$R_{e}$ & 87500 & 112500 & 137500 \\
$\theta^{\prime}$ & 0,050 & 0,084 & 0,127 \\
$\theta_{c}$ & 0,036 & 0,036 & 0,036 \\
$F_{r}$ & 0,22 & 0,28 & 0,35 \\
\hline
\end{tabular}

Le nombre de Reynolds est défini par $R_{e}=U d / v$ avec $U$ la vitesse moyenne du courant et $v$ la viscosité cinématique du fluide $\left(v=1.10^{-6} \mathrm{~m}^{2} / \mathrm{s}\right)$. Le paramètre de Shields sur fond plat est défini tel que $\theta^{\prime}=u_{*}^{\prime} /\left((\mathrm{s}-1) \mathrm{gD} 50\right.$, avec $u_{*}^{\prime}$ la vitesse de frottement au fond dans le cas d'un fond plat et basé sur le diamètre des grains (VAH et al., 2020). Le paramètre de Shields critique pour la mise en mouvement des sédiments, $\theta_{c}$, est estimé en utilisant la formulation de Soulsby (SOULSBY, 1997) : $\theta_{\mathrm{c}}=0,3 /\left(1+1,2 D_{*}\right)+0,055\left(1-\exp \left(-0,02 D_{*}\right)\right)$, avec $D_{*}=((s-1) g / v)^{1 / 3} D_{50}$ et $g$ l'accélération de la gravité. Les valeurs des nombres de Froude $F r=U / \sqrt{g d}<1$ assurent que l'écoulement est de type fluvial.

\section{Description qualitative à l'équilibre}

\section{1 $\underline{\text { Sets M1 et M2 }}$}

La figure 2 montre la couverture sédimentaire à l'équilibre pour des conditions de disponible sédimentaire variant de très limité à illimité pour le set M1. Des structures sédimentaires peuvent être observées à l'équilibre pour tous les tests. La comparaison 


\section{Thème 2 - Dynamique sédimentaire}

visuelle montre de façon évidente que le disponible sédimentaire impacte la morphologie finale $\mathrm{du}$ fond avec une augmentation de la longueur d'onde moyenne des structures quand le disponible sédimentaire augmente.

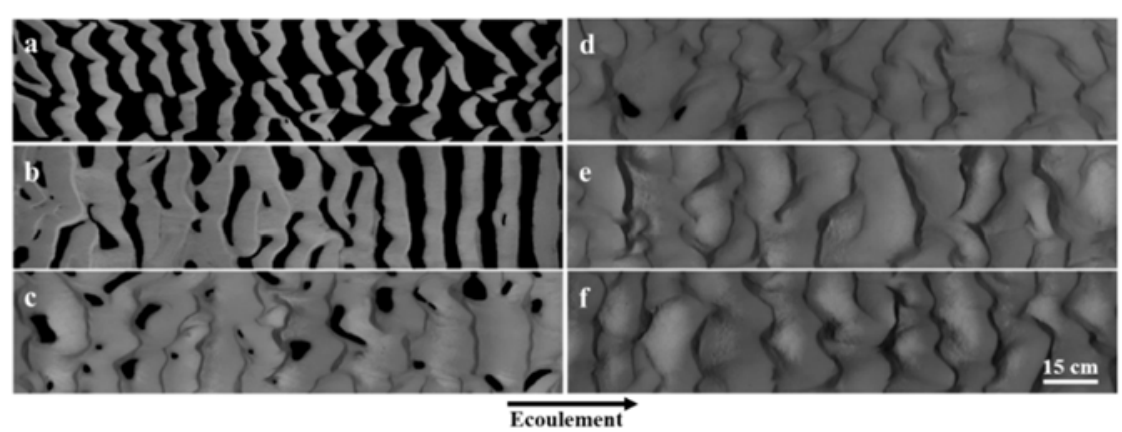

Figure 2. Photos en vue de dessus à l'équilibre pour chaque épaisseur de disponible sédimentaire. Set M1. a) $\delta=0,1 \mathrm{~cm}, b) \delta=0,5 \mathrm{~cm}, c) \delta=1 \mathrm{~cm}, d) \delta=2 \mathrm{~cm}, e) \delta=3,5 \mathrm{~cm}, f$ ) $\delta=5,5 \mathrm{~cm}$. Le fond du canal apparaît en noir.

Trois états de fond sont observés :

- Pour un disponible sédimentaire très limité ( $\delta=0,1 \mathrm{~cm}$ et $0,5 \mathrm{~cm}$; voir figure $2 \mathrm{a}$ et b), les structures sédimentaires sont séparées les unes des autres par des zones non recouvertes de sédiments (en noir sur la figure 2 ) et ce dès les premiers instants de la formation.

- Pour un disponible sédimentaire plus important mais tout de même encore limité ( $\delta=1 \mathrm{~cm}$ et $2 \mathrm{~cm}$; voir figure $2 \mathrm{c}$ et $\mathrm{d}$ ), seules quelques zones non recouvertes sont visibles à l'équilibre. Les structures sédimentaires sont toutefois connectées entre elles.

- Pour les disponibles sédimentaires plus importants $(\delta=3,5 \mathrm{~cm}$ et $5,5 \mathrm{~cm}$; voir figure $2 \mathrm{e}$ et f), l'influence de la limitation du disponible sédimentaire ne peut plus être détectée par une simple observation visuelle du fond.

Les observations effectuées dans le cadre du set M1 sont aussi valables pour le set M2.

\subsection{Set M3}

Le set M3 a été réalisé dans des conditions hydrodynamiques à la limite entre les conditions de formation des rides et des dunes (COLOMBINI \& STOCCHINO, 2011) pour un disponible sédimentaire non limité. A l'équilibre, pour chacun des tests réalisés lors du set M3, un profil bathymétrique a été extrait (voir figure 3). 


\section{XVI'mes Journées Nationales Génie Côtier - Génie Civil \\ Le Havre 2020}

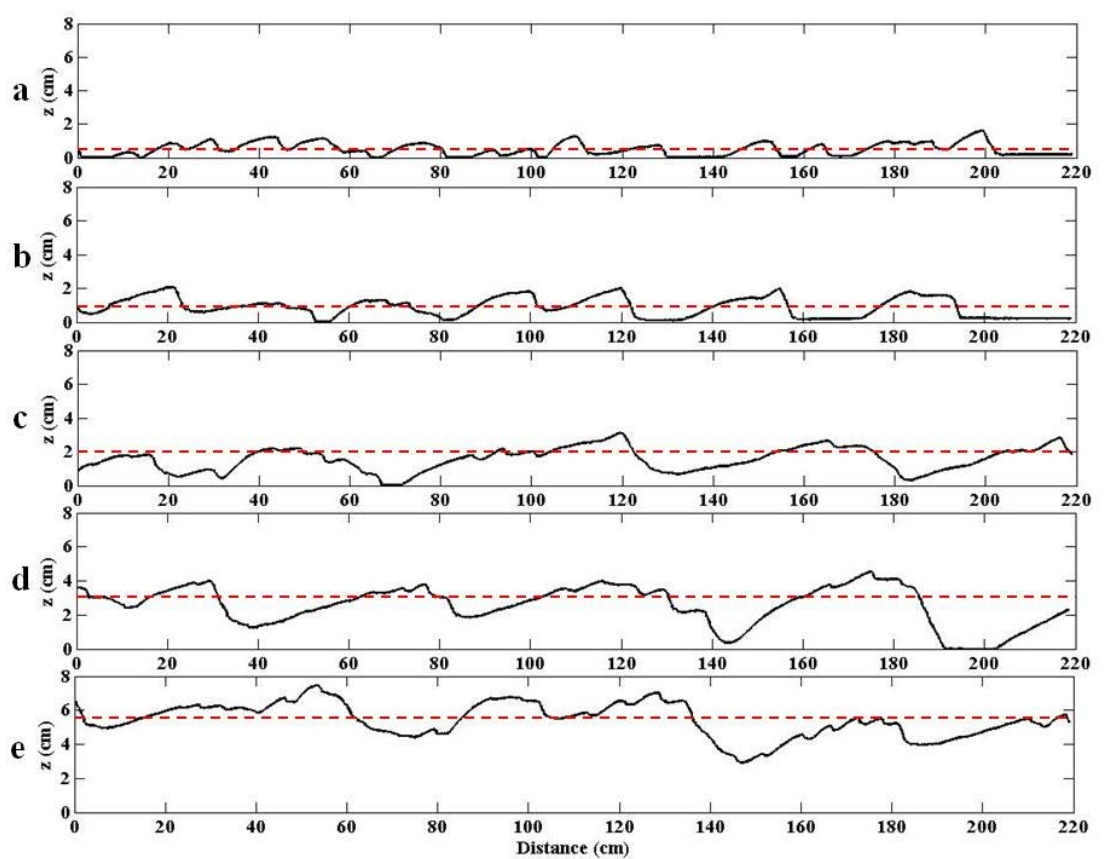

Figure 3. Profils bathymétriques pour le set M3 sur une ligne situé au centre du canal. a) $0,5 \mathrm{~cm}, b) 1 \mathrm{~cm}$, c) $2 \mathrm{~cm}$, d) $3,5 \mathrm{~cm}$ et e) $5,5 \mathrm{~cm}$. Le fond immobile se situe en $\mathrm{z}=0 \mathrm{~cm}$. La ligne rouge en pointillé correspond au niveau initial du disponible sédimentaire.

Dans les cas où le disponible sédimentaire est fortement limité ( $\delta=0,5$ et $\delta=1 \mathrm{~cm}$, voir figure 3 a et $3 b$ ), le fond immobile est présent entre chaque structure sédimentaire. Nous observons aussi une augmentation de la longueur d'onde lorsque le disponible sédimentaire augmente. Ces observations sont comparables avec celles effectuées pour les sets M1 et M2.

Lorsque le disponible sédimentaire devient faiblement limité ou illimité, nous observons l'apparition de structures sédimentaires de petites tailles surimposées à des structures de plus grandes dimensions. Pour un disponible sédimentaire illimité $(\delta=5,5 \mathrm{~cm})$, l'irrégularité des structures sédimentaires semble augmenter comparé aux cas réalisés avec un disponible faiblement limité ( $\delta=2 \mathrm{~cm}$ et $3,5 \mathrm{~cm}$ ). La comparaison visuelle montre que de la même manière que pour les sets $\mathrm{M} 1$ et $\mathrm{M} 2$, le disponible sédimentaire impacte la morphologie finale du fond.

\section{Evolution des longueurs d'onde des structures sédimentaires à l'équilibre en fonction du disponible sédimentaire.}

L'équilibre est atteint quand la moyenne des paramètres géométriques devient invariable dans le temps ou plutôt oscille autour d'une valeur constante. On observe une plus grande variabilité des caractéristiques géométriques pour un disponible illimité que pour un disponible limité. L'écart type à l'équilibre en disponible infini est d'environ $20 \%$ pour les longueurs d'onde alors qu'il est d'environ $5 \%$ en disponible limité. Ce résultat est 


\section{Thème 2 - Dynamique sédimentaire}

aussi visible sur les expériences menées par DREANO et al. (2010). Nous allons considérer l'évolution quantitative des longueurs d'onde des structures sédimentaires à l'équilibre en fonction du disponible sédimentaire.

Une augmentation claire de la longueur d'onde des structures sédimentaires principales est visible quand le disponible sédimentaire augmente (voir figure 4). Par ailleurs, pour la longueur d'onde des structures sédimentaires secondaires surimposées pour le set M3, aucune évolution notable n'est observée.

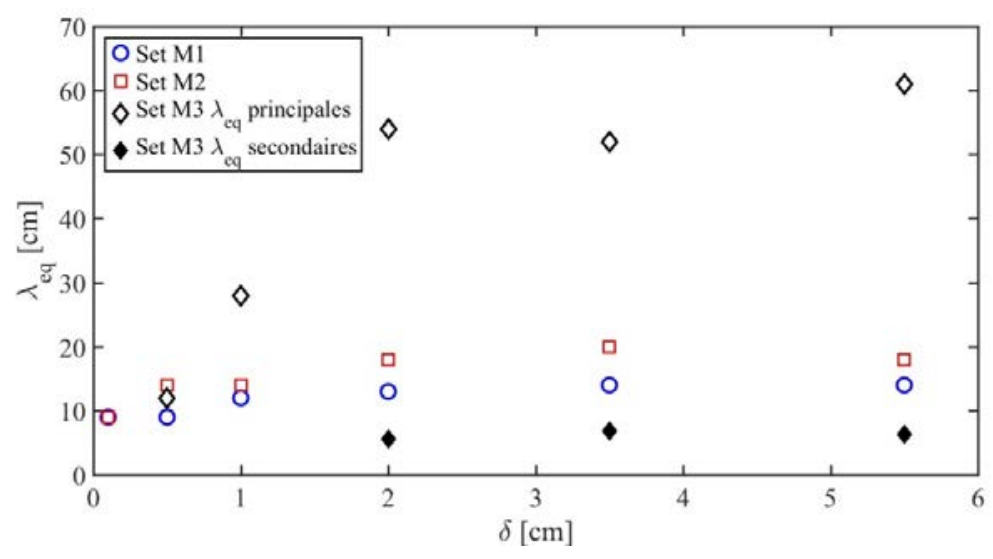

Figure 4. Evolution des longueurs d'onde à l'équilibre pour les sets M1, M2 et M3 en fonction du disponible sédimentaire.

D'après CHAMLEY (1987), les sets M1 et M2 se trouvent dans le domaine des rides de courant et le set M3 dans le domaine des dunes, en disponible infini.

De plus, pour le set M3, les structures petite échelle ne sont visibles de façon continue que pour des conditions de disponible où la couverture sédimentaire est suffisante. En effet, elles n'apparaissent pas pour l'épaisseur la plus faible où la couverture sédimentaire est discontinue $(\delta=0,5 \mathrm{~cm})$ et sont présentes sous forme de patch pour une épaisseur sédimentaire faible $(\delta=1 \mathrm{~cm})$. Ce résultat a aussi été montré par TUIJNDER et al. (2009) qui parle d'un blocage de l'apparition de longueurs d'ondes secondaires quand le disponible sédimentaire est limité. Ce blocage est aussi visible avec l'augmentation de l'irrégularité des structures sédimentaires en disponible faiblement limité.

L'objectif est aussi de comparer les résultats obtenus à l'équilibre des longueurs d'onde

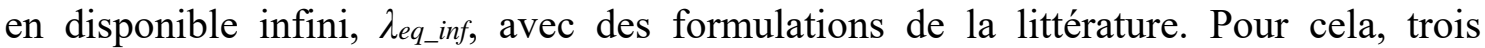
formulations ont été considérées : ALLEN (1968) avec $\lambda_{\text {eq_inf }}=d^{0,6}$ pour la modélisation des dunes, SOULSBY et al. (2012) avec $\lambda_{\text {eq_in }}=D_{50}\left(500+1881 D_{*}^{-1,5}\right)$ où $D_{*}$ est compris entre 1,2 et 16 ainsi que YALIN (1964) avec $\lambda_{\text {eq_inf }}=1000 D_{50}$ dans le cas des rides (voir tableau 2). 


\section{XVIèmes Journées Nationales Génie Côtier - Génie Civil \\ Le Havre 2020}

Tableau 2. Résultats obtenus expérimentalement pour la longueur d'onde à l'équilibre en disponible infini comparés avec trois formulations de la littérature. Pour le set M3, les structures principales et secondaires sont distinguées.

\begin{tabular}{lllll}
\hline Aeq_inf $[\mathrm{m}]$ & Set $\mathbf{M 1}$ & Set $\mathbf{M 2}$ & $\begin{array}{l}\text { Set } \mathbf{M 3} \\
\text { principale }\end{array}$ & $\begin{array}{l}\text { Set } \mathbf{M 3} \\
\text { secondaire }\end{array}$ \\
\hline Mesures de cette étude & 0,14 & 0,18 & 0,56 & 0,06 \\
\hline ALLEN (1968) & 0,44 & 0,44 & 0,44 & 0,44 \\
\% d'écart & $68 \%$ & $59 \%$ & $29 \%$ & $86 \%$ \\
\hline SOULSBY et al. (2012) & 0,19 & 0,19 & 0,19 & 0,19 \\
\% d'écart & $26 \%$ & $5 \%$ & $195 \%$ & $67 \%$ \\
\hline YALIN (1964) & 0,33 & 0,33 & 0,33 & 0,33 \\
\% d'écart & $57 \%$ & $45 \%$ & $71 \%$ & $81 \%$ \\
\hline
\end{tabular}

Les longueurs d'onde à l'équilibre en disponible infini pour les sets M1 et M2 appartiennent au domaine des rides (ASHLEY (1990)) et semblent être mieux modélisées avec la formulation de SOULSBY et al. (2012).

Pour le set M3, d'après les formulations considérées, la longueur d'onde principale en disponible infini semble plus proche du régime des dunes (ALLEN (1968)) que du régime des rides (YALIN (1964); SOULSBY et al. (2012)). En revanche la longueur d'onde secondaire n'est convenablement modélisée ni par la formulation utilisée pour les dunes, ni par celles pour les rides. Les formules prédisent des longueurs d'onde 3 à 6 fois plus grandes que mesurées. Cela confirme que ces formulations sont uniquement prévues pour la prédiction des longueurs d'onde des structures principales.

\section{Conclusions}

Cette étude expérimentale concernant l'étude des longueurs d'ondes des structures sédimentaires à l'équilibre en fonction du disponible sédimentaire, dans le cas d'un forçage hydrodynamique de type courant, a permis d'arriver aux conclusions suivantes :

- En disponible faiblement limité ou illimité, l'irrégularité des structures sédimentaires devient plus importante et des structures secondaires surimposées de petite échelle peuvent apparaître (set M3).

- Pour tous les sets, les longueurs d'onde principales augmentent quand le disponible sédimentaire augmente.

- Les structures sédimentaires des sets M1 et M2 en disponible infini appartiennent au domaine des rides alors que dans le cas du set M3 les structures principales peuvent être considérées comme appartenant au domaine des dunes.

- Un bon accord est trouvé pour la longueur d'onde des structures sédimentaires des sets M1 et M2 en disponible illimité avec la formulation de SOULSBY et al. (2012) et pour les structures principales du set M3 avec la formulation de ALLEN (1968). 


\section{Thème 2 - Dynamique sédimentaire}

- Les longueurs d'onde des structures petite échelle présentes sur les dunes sont beaucoup plus petites que les longueurs d'onde des petites structures de type rides prédites par la littérature.

\section{Références bibliographiques}

ALLEN J. (1968). The nature and origin of bed-form hierarchies. Sedimentology, 10(3), pp 161-182. https://doi.org/10.1111/j.1365-3091.1968.tb01110.x

ASHLEY G.M. (1990). Classification of large-scale subaqueous bedforms: a new look at an old problem. J. Sediment. Petrology, 60(1), pp 160-172. https://doi.org/10.2110/jsr.60.160 CHAMLEY (1987). Sédimentologie. Géosciences, Ed. Dunod, 167 p.

COLOMBINI M., STOCCHINO A. (2011). Ripple and dune formation in rivers. Journal of Fluid Mechanics, 673, pp 121-131. https://doi.org/10.1017/S0022112011000048

DREANO J., VALANCE A., LAGUE D., CASSAR C. (2010). Experimental study on transient and steady-state dynamics of bedforms in supply limited configuration. Earth Surface Processes and Landforms, 35(14), pp 1730-1743. https://doi.org/10.1002/esp.2085

MARIN F., EZERSKY A.B. (2008). Formation dynamics of sand bedforms under solitons and bound states of solitons in a wave flume used in resonant mode. European Journal of Mechanics-B/Fluids, 27(3), pp 251-267. https://doi.org/10.1016/j.euromechflu.2007.05.003 SOULSBY R.L. (1997). Dynamics of marine sands: a manual for practical applications, Ed. Thomas Telford, $249 \mathrm{p}$.

SOULSBY R.L., WHITEHOUSE R. J. S., MARTEN K. V. (2012). Prediction of timeevolving sand ripples in shelf seas. Continental Shelf Research, 38, pp 47-62. https://doi.org/10.1016/j.csr.2012.02.016

TUIJNDER A.P., RIBBERINK J. S., HULSCHER S. J. (2009). An experimental study into the geometry of supply-limited dunes. Sedimentology, 56(6), pp 1713-1727. https://doi.org/10.1111/j.1365-3091.2009.01054.x

VAH M., JARNO A., LE BOT S., FERRET Y., MARIN F. (2020). Bedload transport and bedforms migration under sand supply limitation. Environmental Fluid Mechanics. https://doi.org/10.1007/s10652-020-09738-6

YALIN M.S. (1964). Geometrical properties of sand wave. Journal of Hydraulics Division, 90(5), pp 105-119. 